\title{
An Effective Day Case Treatment Combination for Refractory Neuropathic Mixed Incontinence
}

\author{
Prasad Patki, Joe B Woodhouse, Krishna Patil, Rizwan Hamid, Julian Shah
}

Royal National Orthopaedic Hospital (PP, JBW, JS), Brockley Hill, Stanmore, UK, Institute of Urology and Nephrology (RH, JS), London, UK, Ashford and St. Peters NHS Trust (KP), Chertsey, United Kingdom

\begin{abstract}
Objective: Women with drug refractory neurogenic mixed incontinence (NMI) have limited minimally invasive treatment options and require reconstructive surgery. We examined efficacy of a combination of day case intradetrusor (ID) botulinum toxin (BTX-A) bladder injections and transobturator (TOT) or tension free vaginal tape (TVT).

Materials and Methods: Eleven women who are pharmacotherapy intolerant or who have drug refractory NMI were treated. Two opted for open surgery and the remaining 9 received 1000 units of Dysport diluted in 30 mL saline cystoscopically at 30 ID sites followed by TOT in 6 or TVT in 3 as a day case combination treatment. Patient demographics, pre and post treatment videocystometrogram (VCMG), pad test and International Committee on Incontinence Questionnaire (ICIQ) scores were recorded. At 6 weeks (repeat ICIQ, pad test and patient satisfaction), at 3 and 12 months (VCMG) and 'current' (ICIQ and patient satisfaction) was recorded.

Results: The mean age was 56.7 years (range 41 to 78 ) with a mean follow up of 19.1 months (range 7 to 33 ). All women were continent at 3 and 12 months. Quality of life (ICIQ scores) improved at 6 weeks ( $p>0.001)$ and remained stable up to the last follow up ( $p>0.001)$. Eight women have stopped using pads. At 3 months, there was significant improvement in MDP ( $p>$ $0.014)$ and $\mathrm{MCC}(\mathrm{p}=0.002)$. Anticholinergics were discontinued in 7 with global high satisfaction with the treatment BTXA injections were repeated in 4 (mean 13.5 months).

Conclusion: Anticholinergic refractory women with NMI can be effectively treated as a day case with combination of ID BTX-A injections and TVT or TOT.
\end{abstract}

Key words: incontinence; spinal cord injury; botulinum toxin; sling

Int Braz. J Urol. 2008; 34: 63-72

\section{INTRODUCTION}

Various epidemiological studies based on analysis of postal surveys and interviews using different questionnaires report the prevalence of mixed urinary incontinence (MUI) in the general population in the range varying from $11 \%$ to $61 \%$ with a mean of
$29 \%$ (1). Although there is a lack of clarity and conformity in various definitions of MUI, it may be reasonable to assume that about one third of the cases reporting incontinence suffer from MUI.

Urinary incontinence due to neurological causes is broadly described as is the level of neurological impairment. Classically in suprasacral 
spinal cord injury (SCI), the cause for incontinence is neurogenic detrusor overactivity (NDO) with or without detrusor sphincter dyssynergia (DSD), whereas in lumbosacral spinal injuries, the weakness of the sphincter and pelvic musculature is in question. However, there exists a subpopulation amongst the SCI patients with unknown prevalence that presents with signs and symptoms of combination of urodynamically proven NDO and urodynamic stress incontinence i.e. neurogenic mixed incontinence (NMI).

Traditionally the treatment of MUI targets the more dominant component of the presentation meaning either the urge or the stress. Recently there is a growing body of evidence that anticholinergic medications can be used as a first line of management in urge dominated MUI with improvement in continence rates $(2,3)$. Due to universal need for suppression of the high bladder pressures generated by the NDO, almost all the patients with NMI require anticholinergic medications. When compared to idiopathic detrusor overactivity (IDO), $74 \%$ of NDO patients required higher doses of anticholinergics (4). Intolerance to side effects and non-compliance with pharmacotherapy is reported to be as high as $61 \%$ in patients with NDO (5). Surgical interventions alone (such as suburethral slings or periurethral bulking agents) should be used with caution without effective control over high detrusor pressures $(6,7)$ and may actually be ineffective - particularly in patients with high amplitude bladder contractions (7). In these patients, the only effective treatment options thereafter are sacral anterior root stimulator implant (SARSI) with posterior rhizotomy or CLAM ileocystoplasty and suburethral sling or colposuspension. These major surgical interventions may be unappealing to most patients due to their irreversible nature, morbidity (acute and chronic) and mortality.

Botulinum toxin A (BTX-A) has become an increasingly used treatment option for individuals with either idiopathic or neurogenic detrusor overactivity $(8,9)$. Its injection into the detrusor muscle facilitates a reduction in maximally generated muscle force resulting in increased bladder capacity and compliance, decreased detrusor pressures, the potential to reduce or cease anticholinergic medication, and overall improved continence (8). Being able to effectively control the NDO in female NMI patients with minimally morbid day case procedure allows for the consideration of other treatment options for controlling the pelvic floor weakness component of the NMI. We have previously described the use of BTX-A in a group of patients with neurogenic detrusor overactivity (8) and transvaginal tape in another group of spinal cord injury (SCI) patients with USI (10). We now assess the novel treatment regime of BTX-A combined with a suburethral sling procedure to treat women with refractory neurogenic mixed incontinence secondary to SCI.

\section{MATERIALS AND METHODS}

Starting in April 2003, we identified a total of 22 women with traumatic spinal cord injury who had USI and NDO or loss of compliance (LOC). Initially all women were given extended release oxybutinin (range 20 to $30 \mathrm{mg}$ ) or extended release tolterodine (range 4 to $8 \mathrm{mg}$ ) either alone or in combination. Those women who still experienced bothersome stress incontinence were offered suburethral sling procedures in addition to the pharmacotherapy. Of the 22 women, 11 (50\%) were continent with anticholinergics and sling combination. The remaining 11 women were either intolerant to drugs due to severe side effects like dry mouth, blurring of vision or refractory to the use of anticholinergics - either alone or in combination. They were explained, on the basis of VCMG, that they required treatment for NDO as well as USI. All the options of treatment, like the conservative option (drug combination plus pad usage), minimally invasive day case procedure (combined sling procedure with BTXA) and surgical intervention (combined clam ileocystoplasty and colposuspension) were discussed. The novel nature of the simultaneous use of combined sling procedure with BTX-A was clearly stated to all and the informed consent was obtained in all patients. Apart from two patients who opted for cystoplasty and colposuspension, the remaining 9 women elected for the minimally invasive option involving the day case sling procedure and BTX-A injections. Approval of local Drugs and Therapeutic ethics committee was obtained. 
As a part of the routine pre-operative assessment, an up-to-date (i.e. within 3 months of operation) video-urodynamic evaluation (VCMG) was performed. Parameters assessed included the maximum cystometric capacity (MCC), maximum detrusor pressure (MDP), NDO and the presence of urodynamic stress incontinence (USI). Routine preoperative clinical data recorded included incontinence pad usage, and the current bladder management strategy. Patient quality of life satisfaction was scored with the International Committee for Incontinence Questionnaire (ICIQ). All pre-operative data, i.e. VCMG, ICIQ score, daily leakage record and pad usage, was collected within three months of the day case intervention.

All procedures were undertaken as elective day case procedures. Under propofol anesthesia, BTXA Injections were first given cystoscopically at 30 intradetrusor (ID) sites (as per the technique first described by Schurch et al.), with a total dose of 1000 units of BTX type A (Dysport ${ }^{\circledR}$ ) diluted in $30 \mathrm{~mL}$ of normal saline. Although the dose equivalence between the two preparations (Dysport ${ }^{\circledR}$ and Botox $($ ) ) is variable in the literature, a 1:3 ratio of equivalence between Botox ${ }^{\circledR}$ and Dysport ${ }^{\circledR}$ units respectively was considered acceptable while calculating the total dose of Dysport ${ }^{\circledR}$ BTX A units (i.e. 1000 Dysport ${ }^{\circledR}$ units) (11). Trigone was avoided. Tension free vaginal tape (Gynecare, Ethicon, Johnson \& Johnson, Sommerville, NJ) was applied in 3 women and transobturator tape was passed using the inside out technique (Gynecare TVT-O system, Ethicon, Johnson \& Johnson, Sommerville, NJ) in 6 women. TVT and TOT procedures were discussed with the patients and the final decision was based on patient choice. Regardless of TVT or TOT all patients received cystoscopic checks to rule out bladder trauma. All women were given 120 $\mathrm{mg}$ of gentamycin at the time of induction followed by a week's course of ciprofloxacin. In the 8 women performing intermittent catheterizations (CSIC), a urethral catheter was left in the bladder post-operatively until patients were comfortable to start the CSIC. One patient on suprapubic catheter (SPC) was asked to continue on free drainage for two weeks (time for optimum BTX-A action) and then clamp the catheter regularly to hold volumes up to 350 to $500 \mathrm{~mL}$.
Postoperatively all patients were instructed to cease all anticholinergic medications completely at 2 weeks post surgery as this is the reported time of peak efficacy of the BTX (12). If any patient became incontinent at this point, they had instructions to recommence the pre-surgery dose of anticholinergic treatment and to then titrate themselves off medications until a point was reached where incontinence was controlled with minimum medication. A woman was considered cured when she was satisfied with the procedure and had no demonstrable incontinence associated with either NDO or stress on the VCMG. A routine post-operative follow up was at 6 weeks, at which point a repeat ICIQ assessment was made and the women were asked to record their degree of satisfaction with the procedure. Their choices were: very satisfied, satisfied, neither satisfied nor dissatisfied, and dissatisfied. At this routine follow up, anticholinergic requirement was recorded and VCMG appointments were made for 3 months and then for 1-year post procedure. Thereafter, routine yearly VCMG and Ultrasound surveillance as per standard practice was continued. Instructions were given to patients to contact our service if there were signs of recurrence of NDO (namely either a return of incontinence, decreased bladder capacity, or increasing frequency of intermittent self catheterization) as is our routine protocol for patients who have had BTX-A injections for NDO. At repeat VCMG the parameters recorded were identical to those initially taken. All urodynamic assessment was undertaken both pre- and post-operatively by the same nurse specialist on her routine list and who was disinterested in the novelty of the surgical intervention. To assess ongoing satisfaction/dissatisfaction, all patients were sent an ICIQ and degree of satisfaction choices to complete and return at compilation of this paper. On clinical confirmation of return of storage symptoms and urodynamic confirmation of NDO, repeat BTX-A injections were planned and carried out. The follow up of patients with repeat BTX-A injections was as above.

\section{RESULTS}

All cases were undertaken as planned elective day case surgical treatments. Nine female 
patients have to date undertaken this procedure with a mean age of 56.7 years, standard deviation (SD) 15 years (Table-1). The mean follow up period is currently 19.1 months ( $\mathrm{SD}=8.6$ months). There were no untoward or adverse operative/postoperative outcomes recorded in our cohort. At the initial follow up at six weeks, all nine patients had significantly improved ICIQ scores with 7 out of 9 reporting 'very satisfied' and the remaining two 'satisfied' on satisfaction scale, strongly suggesting excellent patient outcomes. To date the satisfaction scale is unchanged in all and the ICIQ scores remain significantly lower than pre-operative scoring (Table2). All patients attended routine VCMG appointments at 3 months, and seven of these individuals have had VCMG follow up at a year. Maximum follow up is currently 33 months. All 9 patients were continent on VCMG stress testing at 3 months and all 7 were continent at 12 months post-operatively. Urodynamically all 9 patients had improved maximum cystometric capacity (MCC) and reduced maximum detrusor pressure (MDP) with effective suppression of NDO/LOC at 3 months, which was sustained in most at 12 months (Table-3).

To date we have needed to re-inject only 4 patients (patients 1, 2, 7 and 9) with BTX-A for symptoms suggestive of re-emergence of NDO.
Patient 1 has had re-injection twice - firstly at 6 months post initial injection, and then again 14 months later. Patient 2 and 7 have had only a single reinjection at 12 and 17 months post initial injection respectively. Patient 9 has also had two re-injections after an initial interval of 20 months and then 12 months. None of the women has reported any symptoms of hypoasthenia with initial or repeat BTXA injections. At one year, 2 women require preoperative doses of tolterodine for adequate control of NDO. All others have stopped all anticholinergic medications. Although all women no longer feel the need to wear pads, one of them continues to wear a pad a day as an emergency measure (Table-4). Preand post-operative bladder management has remained unchanged (CSIC in 8 and SPC in 1) in all, with none reporting urinary tract infections related to the procedure. Figure-1 shows the treatment algorithm for female neurogenic mixed incontinence.

\section{COMMENTS}

The commonest level of spinal cord injury seen in the adult population is cervical (55\%) with incomplete tetraplegia (13). The thoracolumbar spine is involved in $15 \%$ of injuries and one of the urological

Table 1 - Patient demographics.

\begin{tabular}{lcccccl}
\hline Tag & \multicolumn{2}{c}{ Treatment } & Age (years) & $\begin{array}{c}\text { Length of Follow Up } \\
\text { (months) }\end{array}$ & Injury Level & Spinal Injury \\
& Sling & $\begin{array}{c}\text { BTX } \\
\text { (units) }\end{array}$ & & 24 & L1 & Incomplete \\
\hline 1 & TOT & 1000 & 41 & 14 & L1 & Incomplete \\
2 & TOT & 1000 & 42 & 7 & L3 & Incomplete \\
3 & TOT & 1000 & 62 & 7 & T10 & Complete \\
4 & TOT & 1000 & 47 & 20 & T12 & Incomplete \\
5 & TOT & 1000 & 78 & 24 & T12 & Complete \\
6 & TOT & 1000 & 78 & 19 & T12 & Incomplete \\
7 & TVT & 1000 & 67 & 24 & & Incomplete \\
8 & TVT & 1000 & 51 & 33 & & \\
9 & TVT & 1000 & 44 & 19.1 & & \\
\hline \multicolumn{2}{l}{ Mean } & & 56.7 & 8.6 & & \\
\multicolumn{2}{l}{ Standard deviation }
\end{tabular}

BTX $=$ botulinum toxin $($ Dysport $) ; L=$ lumbar $; T=$ thoracic 


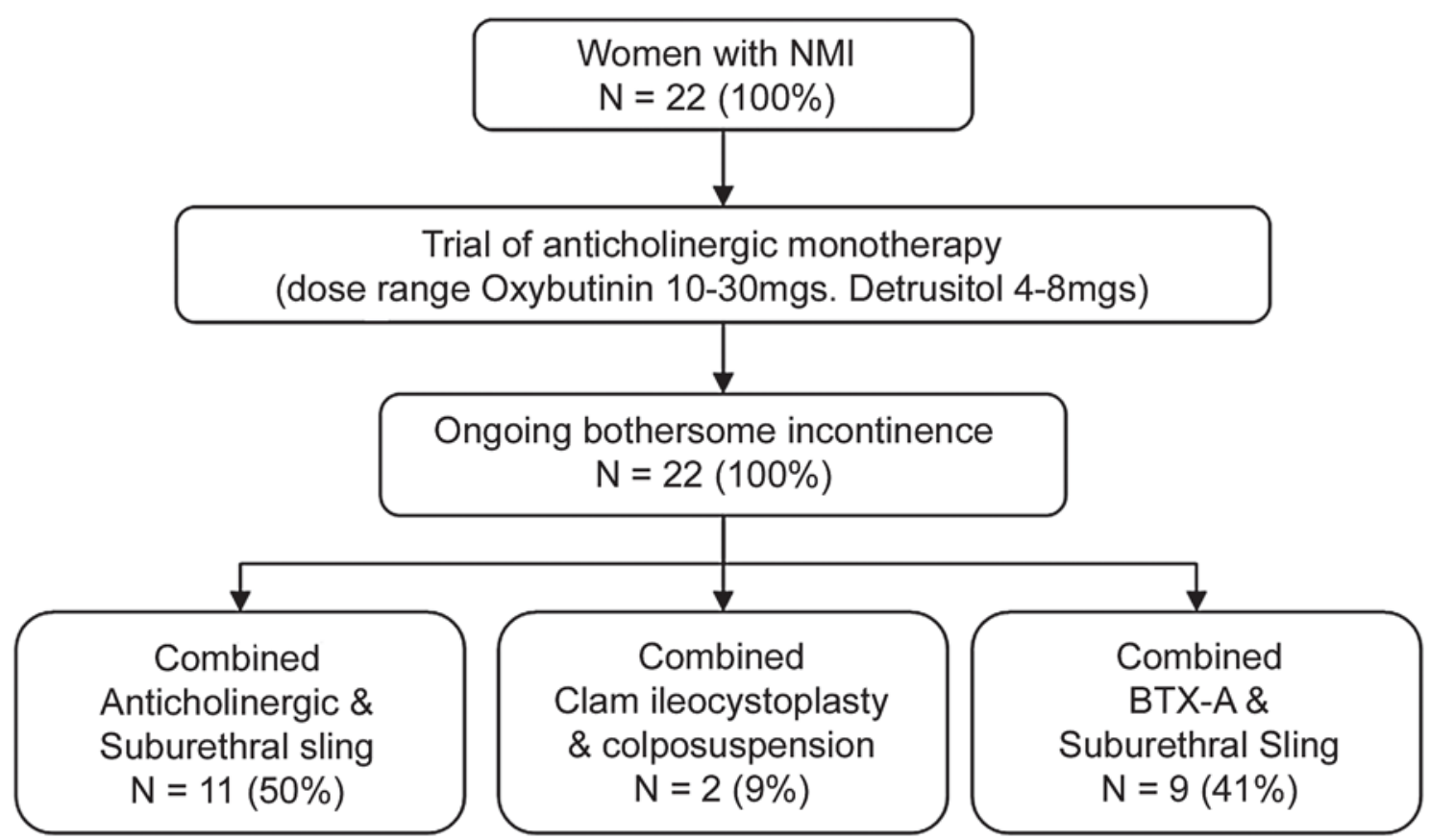

Figure 1 - Treatment algorithm for female neurogenic mixed incontinence.

presentations at this level of injury includes NMI. Although the published literature does not document the prevalence of NMI in the SCI population, they do exist and half of the total numbers of women with NMI are non-responders to pharmacotherapy. In the era of minimally invasive treatments this subset, though

Table 2 - Patient symptom measures pre-and postoperation.

\begin{tabular}{|c|c|c|c|c|c|c|}
\hline \multirow[t]{2}{*}{ Tag } & \multicolumn{3}{|c|}{ ICIQ Quality of Life } & \multicolumn{3}{|c|}{ Urinary Incontinence } \\
\hline & Pre & 6/52 Post & Currently & Pre & 6/52 Post & Currently \\
\hline 1 & 21 & 3 & 0 & All the time & occasional & nil \\
\hline 2 & 21 & 0 & 0 & $>$ 4/day & nil & nil \\
\hline 3 & 18 & 1 & 1 & $>$ 4/day & occasional & occasional \\
\hline 4 & 18 & 0 & 0 & $>$ 4/day & nil & nil \\
\hline 5 & 12 & 0 & 2 & occasional & nil & nil \\
\hline 6 & 16 & 0 & 0 & $>$ 4/day & nil & nil \\
\hline 7 & 17 & 0 & 0 & $>$ 4/day & nil & nil \\
\hline 8 & 17 & 0 & 0 & $>$ 4/day & nil & nil \\
\hline \multirow[t]{5}{*}{9} & 18 & 0 & 0 & All the time & nil & nil \\
\hline & 17.6 & 0.4 & 0.3 & Mean & & \\
\hline & 2.7 & 1.0 & 0.7 & Standard de & & \\
\hline & & 395.0 & 526.7 & Percentage & & \\
\hline & & $>0.001$ & $>0.001$ & $\mathrm{p}$ Value & & \\
\hline
\end{tabular}

$I C I Q=$ International Committee for Incontinence Questionnaire. 
Table 3 - Urodynamic parameters pre- and postoperation.

\begin{tabular}{|c|c|c|c|c|c|c|c|c|c|c|c|c|}
\hline \multirow[t]{2}{*}{ Tag } & \multicolumn{3}{|c|}{ USI } & \multicolumn{3}{|c|}{ MDP } & \multicolumn{3}{|c|}{ MMC } & \multicolumn{3}{|c|}{ NDO } \\
\hline & Pre & $3 / 12$ & $12 / 12$ & Pre & $3 / 12$ & $212 / 12$ & Pre & $3 / 12$ & $12 / 12$ & Pre & 3/12 Post & $12 / 12$ \\
\hline 1 & $\mathrm{Y}$ & $\mathrm{N}$ & $\mathrm{N}$ & 33 & 16 & 51 & 110 & 420 & 422 & $\mathrm{Y}$ & $\mathrm{N}$ & $\mathrm{Y}$ \\
\hline 2 & $\mathrm{Y}$ & $\mathrm{N}$ & $\mathrm{N}$ & 34 & 10 & 8 & 318 & 450 & 500 & Y & $\mathrm{N}$ & $\mathrm{N}$ \\
\hline 3 & $\mathrm{Y}$ & $\mathrm{N}$ & - & 58 & 12 & - & 141 & 400 & - & Y & $\mathrm{N}$ & - \\
\hline 4 & $\mathrm{Y}$ & $\mathrm{N}$ & - & 50 & 8 & - & 420 & 600 & - & $\mathrm{Y}$ & $\mathrm{N}$ & - \\
\hline 5 & $\mathrm{Y}$ & $\mathrm{N}$ & $\mathrm{N}$ & 20 & 17 & 11 & 180 & 188 & 210 & LOC at 88 & 8 LOC at 168 & 3 LOC at 105 \\
\hline 6 & $\mathrm{Y}$ & $\mathrm{N}$ & $\mathrm{N}$ & 38 & 18 & 22 & 260 & 450 & 390 & $\mathrm{Y}$ & $\mathrm{N}$ & $\mathrm{Y}$ \\
\hline 7 & Y & $\mathrm{N}$ & $\mathrm{N}$ & 93 & 20 & 14 & 500 & 700 & 640 & $\mathrm{Y}$ & Y & $\mathrm{N}$ \\
\hline 8 & $\mathrm{Y}$ & $\mathrm{N}$ & $\mathrm{N}$ & 74 & 42 & 40 & 225 & 412 & 510 & $\mathrm{Y}$ & $\mathrm{N}$ & $\mathrm{Y}$ \\
\hline \multirow[t]{5}{*}{9} & $\mathrm{Y}$ & $\mathrm{N}$ & $\mathrm{N}$ & 46 & 15 & 7 & 350 & 625 & 600 & $\mathrm{Y}$ & $\mathrm{N}$ & $\mathrm{N}$ \\
\hline & & & Mean & 49.6 & 17.6 & 21.9 & 278.2 & 471.7 & 467.4 & & & \\
\hline & Sta & dard de & eviation & 22.6 & 10.0 & 17.2 & 130.4 & 152.0 & 144.1 & & & \\
\hline & & Percent & t change & & 282.3 & 226.7 & & 59.0 & 59.5 & & & \\
\hline & & & $\mathrm{p}$ Value & & 0.014 & 0.06 & & 0.002 & 0.002 & & & \\
\hline
\end{tabular}

$M M C=$ maximum cystometric capacity $; M D P=$ maximum detrusor pressure $;$ USI = urodynamic stress incontinence; NDO = neurogenic detrusor overactivity; $T V T=$ transvaginal tape; $T O T=$ transobturator tape $;$ LOC $=$ loss of compliance; BTX = botulinum toxin

small in number, poses a difficult clinical challenge, which is addressed by this simultaneous multimodal treatment.

The near universal 'best management' practice of intermittent catheterization in SCI individuals with NDO means that issues to do with retention were not present. This would certainly need to be discussed with patients with idiopathic MUI. In SCI individuals, only treating the NDO or pelvic floor weakness is insufficient to render these patients dry. Similarly in this group without control over the NDO, patients will remain wet. Use of BTX-A injections as

Table 4 - Patient clinical management data pre- and postoperation.

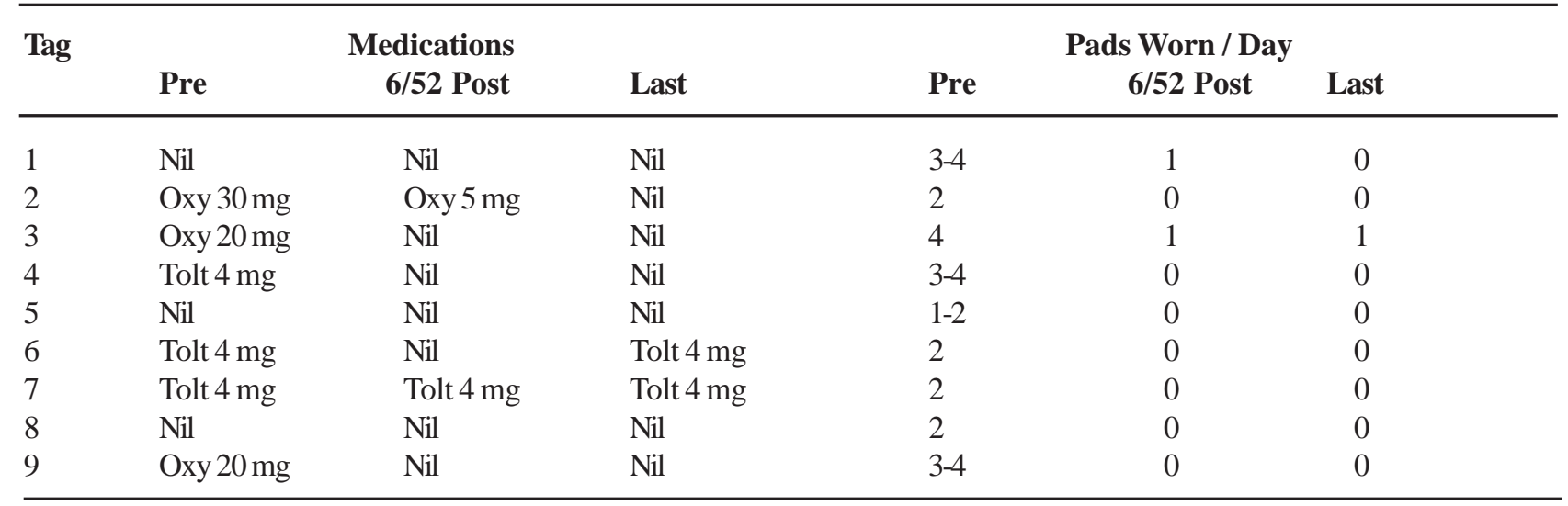

Oxy $=$ oxybutinin $;$ Tolt $=$ tolterodine $; m g=$ milligram . 
a single modality treatment in NMI patients was unsatisfactory. Although the hyperreflexia was well controlled, the patients still remained wet and scored poorly on the ICIQ (8). Hence we have elected for simultaneous treatment of both the stress and urge components. We have not yet observed any complications arising from the use of intermittent catheterization related to the increased tightness of the urethra in our cohort, and sling procedures are acceptably used in SCI patients (e.g. for acontractile bladders with stress incontinence) with no issue (10).

It is interesting to note that 2 women opted for a permanent surgical option of CLAM ileocystoplasty and colposuspension. This may be due to the need for repeat, BTX-A injections every 9 to 12 months. Our data is too sparse to draw any firm conclusions regarding this; however the re-injection rates seen in our group are currently ranging between 6 - 20 months. The MDP change at 12 months was not statistically significant $(\mathrm{p}=0.06)$ and MDP actually increased in one of the patients. This may represent a waning toxin action on the bladder and increased infravesical resistance due to TOT. However, the MCC and continence was maintained. Likewise the low preoperative MDP (<40 cm water) seen in some women may be due to the combination of pre-operative anticholinergic intake (patient no. 2 and 6), loss of compliance at low volumes leading to early leakage (patient no. 5), and an NDO at low bladder capacity leading to early leakage in addition to VCMG documented USI. Similarly the large bladder capacity in some may reflect the pre-operative intake of maximum tolerated doses of anticholinergics.

Similarly two other patients have recurrent NDO at 12 months but remain continent at a lower MDP (Table-3). Since the recurrence of NDO may clinically present as increased frequency of CSIC with reduced volumes and objectively as increased MDP with or without incontinence, a regular VCMG study seems to be mandatory in these patients. In SCI women with NMI the re-treatment with BTX-A depends equally on the clinical recurrence of symptoms and the sustained rise in MDP levels. The concern about less than satisfactory cure rates of surgery in presence of detrusor overactivity (14) and development of de novo urgency seen in up to $26 \%$ patients after mid urethral sling procedure (15) are non issues with this combination treatment in women with NMI.

The authors acknowledge that there are reservations relating to the use of short form questionnaires relating to their intrinsic subjectivity and that the ICIQ has not been specifically validated for use with SCI patients. However, it has been validated as a simple, sensitive, and reliable assessment of the impact on quality of life of urinary incontinence. The improved follow up ICIQ scores ( $p>0.001$ at 6 weeks and current) and satisfaction with treatment at 6 weeks and finally at the time of compilation of this article reflect strongly the improvement in the individual's experience of continence during this period. The recording of pads worn per day is also open to subjective variability as a measure of continence. However, when patients stop wearing pads altogether, it is still a useful measure reflecting a significant improvement in continence in all but one in this group.

All urodynamic testing was only undertaken by a single observer who, although not formally blinded to the procedure, was unaware of the novel nature of this intervention, and testing was undertaken as a routine post procedure or routine yearly follow up. We feel that because of this, no systematic influence was present on the urodynamic findings. Equally it was not possible to blind the operating surgeon to the procedure being undertaken and so it is possible that the operative procedure could have resulted in 'tight slings' that might be thought to bias towards almost $100 \%$ continence rates (Table- 2 and 3 ).

The use of BTX-A in the management of urinary incontinence is gaining popularity amongst both clinicians and specifically patients who can become free of the side effects and bother of daily medications. On a cautionary note, however, there is a lack of data pertaining to the long term sequelae of the usage of BTX when given into the detrusor. Hafarkamp et al. observed no significant histopathological changes in the detrusor muscle biopsied before and after BTX injections (up to 22 months following injection) (16). It is known that some individuals can develop an immune mediated tolerance to BTX-A with repeated exposure and this may eventually lead to late failures seen with this procedure. Salvage in this circumstance 
might be possible with BTX type B, which has different immunogenisity (17).

The ability to undertake treatment as a day case is undoubtedly a popular aspect of this procedure with our patients. Follow up injections of BTX-A are required but are conducted on a day case basis. The ongoing cost implications of this treatment have still to be fully assessed. BTX-A injections (per treatment) have been calculated at costing $\leq 846$ when using flexible cystoscopy and as an outpatient (18). Also, there is the extra workload burden for the urologist undertaking repeated procedures that needs to be factored in. We feel that allowing the need for patient autonomy and choice, the lack of major morbidity and mortality; and the presented clinical efficacy of this intervention mean that this combination therapy certainly warrants further assessment in a larger multicenter study.

\section{CONCLUSION}

The combined suburethral sling (TVT/TOT) and intra-detrusor Botulinum toxin A therapy is a novel and efficacious day case intervention. In this pilot study we have achieved excellent success at restoration of continence in female SCI patients with mixed incontinence that are intolerant of medical treatments. Based on short term results and patient satisfaction with this procedure in this small group of women, it is safe to say that SCI women with NMI now have an alternative treatment option that does not involve major and disfiguring surgery.

\section{CONFLICT OF INTEREST}

None declared.

\section{REFERENCES}

1. Hunskaar S, Burgio K: Epidemeology of Urinary and Faecal Incontinence and Pelvic Organ Prolapse. In: Abrams P, Cardozo L, Khoury S (ed.), Incontinence. Paris, Health Publication. 2005; pp. 255-312.
2. Staskin DR, Te AE: Short- and long-term efficacy of solifenacin treatment in patients with symptoms of mixed urinary incontinence. BJU Int. 2006; 97: 125661.

3. Wein AJ: Treatment of urge-predominant mixed urinary incontinence with tolterodine extended release: a randomized, placebo-controlled trial. J Urol. 2005; 173: 2056-7.

4. Bennett N, O'Leary M, Patel AS, Xavier M, Erickson JR, Chancellor MB: Can higher doses of oxybutynin improve efficacy in neurogenic bladder? J Urol. 2004; 171: 749-51.

5. Szollar SM, Lee SM: Intravesical oxybutynin for spinal cord injury patients. Spinal Cord. 1996; 34: 284-7.

6. P. Abrams, L. Cardozo, S. Khoury, A. Wein, In: Incontinence: 2nd International Consultation on Incontinence July 1-3. Paris, Health Publications. 2001; p. 513.

7. Schrepferman CG, Griebling TL, Nygaard IE, Kreder KJ: Resolution of urge symptoms following sling cystourethropexy. J Urol. 2000; 164: 1628-31.

8. Patki PS, Hamid R, Arumugam K, Shah PJ, Craggs M: Botulinum toxin-type A in the treatment of drugresistant neurogenic detrusor overactivity secondary to traumatic spinal cord injury. BJU Int. 2006; 98: 7782.

9. Popat R, Apostolidis A, Kalsi V, Gonzales G, Fowler CJ, Dasgupta P: A comparison between the response of patients with idiopathic detrusor overactivity and neurogenic detrusor overactivity to the first intradetrusor injection of botulinum-A toxin. J Urol. 2005; 174: 984-9.

10. Hamid R, Khastgir J, Arya M, Patel HR, Shah PJ: Experience of tension-free vaginal tape for the treatment of stress incontinence in females with neuropathic bladders. Spinal Cord. 2003; 41: 118-21.

11. Ranoux D, Gury C, Fondarai J, Mas JL, Zuber M: Respective potencies of Botox and Dysport: a double blind, randomised, crossover study in cervical dystonia. J Neurol Neurosurg Psychiatry. 2002; 72: 45962.

12. Schurch B, de Seze M, Denys P, Chartier-Kastler E, Haab F, Everaert K, et al.: Botulinum toxin type a is a safe and effective treatment for neurogenic urinary incontinence: results of a single treatment, randomized, placebo controlled 6-month study. J Urol. 2005; 174: 196-200.

13. Sekhon LH, Fehlings MG: Epidemiology, demographics, and pathophysiology of acute spinal cord injury. Spine. 2001; 26(Suppl 24): S2-12. 
14. Colombo M,Zanetta G, Vitobello D, Milani R:^The Burch colposuspension for women with and without detrusor overactivity. Br J Obstet Gynaecol. 1996; 103: 255-60.

15. Jeffry L, Deval B, Birsan A, Soriano D, Darai E: Objective and subjective cure rates after tension-free vaginal tape for treatment of urinary incontinence. Urology. 2001; 58: 702-6.

16. Haferkamp A, Schurch B, Reitz A, Krengel U, Grosse J, Kramer G, et al.: Lack of ultrastructural detrusor changes following endoscopic injection of botulinum toxin type a in overactive neurogenic bladder. Eur Urol. 2004; 46: 784-91.
17. Ghei M, Maraj BH, Miller R, Nathan S, O'Sullivan C, Fowler CJ, et al.: Effects of botulinum toxin B on refractory detrusor overactivity: a randomized, doubleblind, placebo controlled, crossover trial. J Urol. 2005; 174: 1873-7; discussion 1877.

18. Kalsi V, Popat RB, Apostolidis A, Kavia R, Odeyemi IA, Dakin HA, et al.: Cost-consequence analysis evaluating the use of botulinum neurotoxin-A in patients with detrusor overactivity based on clinical outcomes observed at a single UK centre. Eur Urol. 2006; 49: 519-27.
Accepted after revision: September 26, 2007

\section{Correspondence address:}

Dr. Prasad Patki

Department of Neurourology

London Spinal Cord Injuries Centre

RNOH Trust

Brockley Hill, Stanmore, HA7 4LP, UK

Fax: + 440208 909-5343

E-mail:prasadpatki@hotmail.com

\section{EDITORIAL COMMENT}

The authors are commended on a nice review of a small number of patients with neurogenic mixed stress and urge urinary incontinence who underwent concomitant sling and botulinum toxin A (BTX-A) injection of the detrusor muscle. Because of reports of promising early results, the use of BTX-A injection for treatment of urgency refractory to pharmacologic therapy has recently started to gain popularity in spite of the fact that it is yet to be FDA-approved in the United States for this specific indication. Accordingly, the use of intradetrusor BTX-A in general remains in its infancy, but this group interestingly reports on its use in a neurogenic population who has mixed incontinence.
Several thoughts came to mind as this manuscript was reviewed. It is typically our practice to get the urgency component under as good control as possible prior to placing a sling in the mixed urinary incontinence (MUI) patient. This thinking has changed slightly as evidence that the midurethral slings, particularly those placed via the transobturator approach may have a lower tendency to cause or exacerbate urgency symptoms. Indeed, there have been reports of resolution of urgency in MUI patients following midurethral slings in several series. It would be interesting to discuss those patients in whom urgency persists or worsens following simultaneous BTX-A injection and sling and what options would be 
considered for treating the urgency in those patients postoperatively.

Finally, "Cure was defined when women were satisfied with the procedure and had no demonstrable incontinence associated with either NDO or stress on the VCMG." Were women not considered cured if they had any leakage even if they were satisfied? In this day and age of emphasis on quality of life parameters and patient goals, perhaps outcomes of anti-incontinence procedures can be considered positive even if some urinary leakage persists. This manuscript was nicely written and I look forward to longer follow up in this and other groups receiving BTX-A.

Dr. Kathleen C. Kobashi

Head, Section of Urology \& Renal Transplantation Virginia Mason Medical Center

Seattle, Washington, USA

E-mail:urokck@vmmc.org

\section{REPLY BY THE AUTHORS}

Although we agree with reviewer's practice of getting storage symptoms under control prior to anti-incontinence procedure the BTX-A injection results were very promising. In $76 \%$ of the patients BTX-A injections resolve neurogenic detrusor overactivity (NDO) and in the remaining $24 \%$ there is a reduction in maximum detrusor pressure (1). This reduced reflex activity is then more amenable for control with the reintroduction of anticholinergics. In our experience of over 150 patients on BTX-A treatment around $86 \%$ remain continent with reduced or no anticholinergic medications (unpublished data). These results prompted us to treat both the components of neurogenic mixed incontinence simultaneously.

We assess the bladder activity objectively by videourodynamics at 3 months and would offer a repeat dose of BTX-A if symptoms were to persist. Failing these remedies, the options would be CLAM ileocystoplasty or Sacral Anterior Root Stimulator Implant with posterior rhizotomy.

The reports of resolution of urge, post the sling procedure are mostly limited to idiopathic mixed urinary incontinence (MUI) and would be unlikely in
NMI. Indeed in patients with NMI who were treated with only BTX-A injections the stress incontinence persisted and remained bothersome in all (1). These patients were subsequently treated with suburethral sling procedure.

The quality of life (QOL) is indeed an important end point in studies involving patients with incontinence. In MUI population urgency incontinence is reported to be a more bothersome symptom compared to stress incontinence and resolution of urgency incontinence positively affects the QOL. However, in the spinal cord injured women incontinence ('firing off') is a primary complaint, followed by urgency and frequency. In this small subset we considered objectively proven continence as cure and not surprisingly the satisfaction scale mirrored the post treatment continence status.

\section{REFERENCE}

1. Patki PS, Hamid R, Arumugam K, Shah PJ, Craggs M: Botulinum toxin-type $\mathrm{A}$ in the treatment of drugresistant neurogenic detrusor overactivity secondary to traumatic spinal cord injury. BJU Int. 2006; 98: 77-82. 